Heléne E.K. Sundelin, MD

Henrik Larsson, $\mathrm{PhD}$

Paul Lichtenstein, PhD

Catarina Almqvist, $\mathrm{PhD}$

Christina M. Hultman,

$\mathrm{PhD}$

Torbjörn Tomson, $\mathrm{PhD}$

Jonas F. Ludvigsson, PhD

Correspondence to

Dr. Sundelin:

helene.sundelin@hotmail.com

Editorial, page 130

Supplemental data at Neurology.org

\section{Autism and epilepsy}

\section{A population-based nationwide cohort study}

[미

\section{ABSTRACT}

Objective: To investigate the risk of autism spectrum disorder (ASD) in individuals with epilepsy and in their first-degree relatives to determine shared etiology.

Methods: Through the Swedish Patient Register, we identified 85,201 individuals with epilepsy, as well as all their siblings ( $n=80,511)$ and offspring $(n=98,534)$. Each individual with epilepsy was compared with 5 controls, matched for age, sex, calendar period, and county, while siblings and offspring were compared with siblings and offspring of controls. We excluded siblings and offspring with epilepsy. Using Cox regression, we calculated hazard ratios (HRs) for future diagnosis of ASD. Logistic regression was applied to calculate odds ratios (ORs) for prior diagnosis of ASD.

Results: During follow-up, 1,381 (1.6\%) individuals with epilepsy and $700(0.2 \%)$ controls were diagnosed with ASD. Individuals with epilepsy were therefore at increased risk of future ASD (HR 10.49, 95\% confidence interval [Cl] 9.55-11.53), with the highest risk seen in individuals diagnosed with epilepsy in childhood. Both siblings (HR 1.62, 95\% Cl 1.431.83) and offspring (HR 1.64, 95\% Cl 1.46-1.84) of epilepsy patients were at increased risk of ASD. The risk in the offspring was particularly high in mothers with epilepsy (HR 1.91; 95\% $\mathrm{Cl} 1.63-2.23)$. Epilepsy was also associated with a prior diagnosis of ASD (OR $4.56,95 \% \mathrm{Cl}$ 4.02-5.18).

Conclusions: Individuals with epilepsy are at increased risk of ASD, especially if epilepsy appears in childhood. Further, ASD is more common in the siblings and offspring of individuals with epilepsy, suggesting shared etiology. Neurology ${ }^{\circledR} 2016 ; 87: 192-197$

\section{GLOSSARY}

ASD = autism spectrum disorder; $\mathbf{C l}=$ confidence interval; $\mathbf{H R}=$ hazard ratio; ICD = International Classification of Diseases; $\mathbf{O R}=$ odds ratio.

According to the definition of the International League Against Epilepsy, "epilepsy is a disorder of the brain characterized by an enduring predisposition to generate epileptic seizures and by the neurobiologic, cognitive, psychological, and social consequences of this condition." ${ }^{1}$ Epilepsy frequently co-occurs with psychiatric disturbances, such as mood and anxiety disorders and autism spectrum disorder (ASD). ${ }^{1}$

ASD is a neurodevelopmental disorder characterized by impairment in social interaction and communication and restricted, repetitive, or stereotypic behavior, interests, and activities. ${ }^{2}$

The strength and nature of the association between epilepsy and ASD have been scarce and difficult to interpret. A US study of health care data, a British psychiatric morbidity survey, and a prospective screening study all found that ASD as a comorbidity in patients with epilepsy had a prevalence between $1.3 \%$ and $21 \%$ and an odds ratio (OR) between 6 and $22 .{ }^{3-5}$ Prevalence of epilepsy in ASD has been studied in different large cohorts with results varying from $3.9 \%$ to $19.4 \% .^{6-9}$

From the Department of Pediatrics (H.E.K.S.), University Hospital, Linköping; Departments of Medical Epidemiology and Biostatistics (H.L., P. L., C.A., C.M.H., J.F.L.) and Clinical Neuroscience (T.T.), Karolinska Institutet; Astrid Lindgren Children's Hospital (C.A.), Karolinska University Hospital, Stockholm; Department of Pediatrics (J.F.L.), University Hospital, Örebro, Sweden; and Division of Epidemiology and Public Health (J.F.L.), School of Medicine, University of Nottingham, UK.

Go to Neurology.org for full disclosures. Funding information and disclosures deemed relevant by the authors, if any, are provided at the end of the article. 
This study estimated the risk of ASD in individuals with epilepsy and their relatives in order to explore common risk factors. We hypothesized that individuals with epilepsy and their relatives would be at increased risk of ASD.

METHODS We used the Swedish Patient Register ${ }^{10}$ to identify all individuals with epilepsy, defined according to the International Classification of Diseases (ICD). Status epilepticus and febrile convulsions were not included in our definition of epilepsy (ICD-7: 353, except for 353.2; ICD-8: 345, except for 345.2; ICD-9: 345, except for 345Q; ICD-10: G40). The Swedish Patient Register includes inpatient diagnoses from parts of Sweden since 1964 (nationwide since 1987) and hospital-based outpatient care since 2001. ${ }^{10}$

We identified siblings, offspring, and parents of all patients with epilepsy. For our analyses, we excluded all relatives and controls with epilepsy. We analyzed relatives of patients with epilepsy and relatives of patients with epilepsy but without ASD.

For each individual with epilepsy, we identified up to 5 controls matched for age, sex, year of birth, and county of residence at epilepsy diagnosis using the Swedish Total Population Register. ${ }^{11}$

For initial analyses, we excluded anyone with an ASD diagnosis prior to the diagnosis of epilepsy or corresponding date in matched reference individuals (study entry). We estimated the risk of ASD in the siblings and offspring of index individuals with epilepsy and then the risk of ASD in the siblings and offspring of index individuals with epilepsy but no ASD.

ASD was defined as having relevant ICD codes in the Swedish National Patient Register since 1987 (when ICD-9 started in Sweden): ICD-9: 299A, 299B, 299W, 299X; ICD-10: F84.0F84.5, F84.8, and F84.9.

Data on ASD were linked to epilepsy using the unique personal identity number ${ }^{11}$ assigned to all Swedish residents.

Follow-up started on the date of the epilepsy diagnosis (and corresponding date in matched controls) and ended with ASD diagnosis, death, or emigration, or on December 31, 2009, whichever occurred first. For offspring, siblings, parents, and their controls, the observation time started in 1987 or with their birth

\section{Table 1 Description of patients with epilepsy and controls}

\begin{tabular}{|c|c|c|}
\hline & Epilepsy & Controls \\
\hline Individuals, $\mathrm{n}$ & 85,201 & 425,760 \\
\hline Male, n (\%) & $45,120(53.0)$ & 225,427 (52.9) \\
\hline Female, $n(\%)$ & $40,081(47.0)$ & $200,333(47.1)$ \\
\hline Age at diagnosis of epilepsy, y, median (range) & $44.8(0.1-99.3)$ & $44.8(0.1-99.3)^{a}$ \\
\hline \multicolumn{3}{|l|}{ Age at study entry, $y, n(\%)$} \\
\hline Less than 19 & $22,993(27.0)$ & $114,806(27.0)$ \\
\hline 20-39 & 15,556 (18.3) & 77,724 (18.3) \\
\hline $40-59$ & $18,289(21.5)$ & $91,421(21.5)$ \\
\hline 60 or greater & 28,289 (33.3) & 141,809 (33.3) \\
\hline \multicolumn{3}{|l|}{ Date of first diagnosis with epilepsy, ${ }^{a}$ n (\%) } \\
\hline $1973-1986$ & $15,736(18.5)$ & $78,680(18.5)$ \\
\hline 1987-1996 & 22,568 (26.5) & $112,833(26.5)$ \\
\hline $1997-2009$ & $46,897(55.0)$ & $234,247(55.0)$ \\
\hline
\end{tabular}

${ }^{\text {a } A n d ~ c o r r e s p o n d i n g ~ d a t e ~ i n ~ m a t c h e d ~ c o n t r o l s . ~}$ and ended with a diagnosis of ASD, death, or emigration, or on December 31, 2009.

In separate analyses we stratified for sex, age at first registered diagnosis of epilepsy (0-19, 20-39, 40-59, and $\geq 60$ years), and calendar period (1986, 1987-1996, and 1997). Analyzing the risk of ASD in the siblings and offspring of individuals with epilepsy, we stratified for the same variables as in the separate analyses but also for sex of the index person.

Cox regression was used to estimate hazard ratios (HRs) for future ASD in individuals with epilepsy and in their first-degree relatives. Our statistical model was internally stratified, i.e., an individual with epilepsy was only compared with his or her agematched controls for ASD. The overall HR presented in this article is a summary from risk estimates calculated for each stratum (consisting of 1 index individual and up to 5 controls). To test the proportional hazards assumptions, log-minus-log curves were plotted.

In other separate analyses we also examined the association between epilepsy and ASD when 2 diagnoses of both exposure (epilepsy) and outcome measure (ASD) were required.

Using conditional logistic regression, we also examined the risk of ASD prior to epilepsy.

Statistical analyses were conducted using SPSS software (version 18; SPSS, Inc., Chicago, IL). Relative risks with $95 \%$ confidence intervals $(\mathrm{CIs})$ that did not include 1.0 were regarded as statistically significant.

Standard protocol approvals, registrations, and patient consents. The study was approved by the Regional Ethics Committee, Stockholm, Sweden (protocol no. 2009/5:10), which adjudicated that individual informed consent was not required. ${ }^{12}$

RESULTS Initially, 86,916 individuals with epilepsy and 434,580 matched controls were identified. After exclusion of study participants with a prior diagnosis of ASD, the final sample comprised 85,201 individuals with epilepsy and 425,760 controls (table 1).

The median follow-up between epilepsy (or study entry in matched controls) and ASD diagnosis was 5.5 years (range 0-36.3 years) for individuals with epilepsy and 6.1 years for controls (range 0-36.0 years). For individuals with epilepsy, the median age at the first diagnosis of ASD was 13.6 years (range 0.6-73.6 years) and 17.5 years for controls (range 2.2-90.9 years).

During follow-up, 1,381 (1.6\%) individuals with epilepsy and $700(0.2 \%)$ controls were diagnosed with ASD (table 2). Thus, individuals with epilepsy were at increased risk of future ASD (HR 10.49, 95\% CI 9.55-11.53). The risk of ASD was increased in both male (HR 9.27, 95\% CI 8.25-10.41) and female patients with epilepsy (HR 13.10, 95\% CI 11.15-15.39) (table 2). The difference in HRs between male and female patients was statistically significant ( $p$ value for interaction between epilepsy and sex was $<0.001)$.

The risk of future ASD was especially high in individuals diagnosed with epilepsy in childhood (aged $<20$ years, table 2) (HR 11.39, 95\% CI 10.26-12.64, $p$ value for interaction between epilepsy and age $<0.001$ ). 
Table 2 Future risk of autism spectrum disorder (ASD) in individuals with epilepsy

\begin{tabular}{|c|c|c|c|}
\hline & $\begin{array}{l}\text { No. }(\%) \text { of ASD cases in } \\
\text { patients with epilepsy }\end{array}$ & $\begin{array}{l}\text { No. }(\%) \text { of ASD cases } \\
\text { in controls }\end{array}$ & HR (95\% Cl) \\
\hline All & 1,381/85,201 (1.6) & $700 / 425,760(0.2)$ & $10.49(9.55-11.53)^{a}$ \\
\hline Male & $830 / 45,120$ (1.8) & 485/225,427 (0.2) & $9.27(8.25-10.41)^{a}$ \\
\hline Female & $551 / 40,081$ (1.4) & 215/200,333 (0.1) & $13.10(11.15-15.39)^{a}$ \\
\hline $\operatorname{Age}^{a}$ 0-19 y & 1,187/22,993 (5.2) & $555 / 114,806(0.5)$ & $11.39(10.26-12.64)^{a}$ \\
\hline $\operatorname{Age}^{a} 20-39 y$ & $155 / 15,556$ (1.0) & $108 / 77,724(0.1)$ & $7.34(5.70-9.44)^{a}$ \\
\hline $\operatorname{Age}^{a} 40-59$ y & $35 / 18,289$ (0.2) & $25 / 91,421(<0.10)$ & $8.00(4.66-13.76)^{a}$ \\
\hline Age $\geq 60 y^{b}$ & $4 / 28,363(<0.01)$ & $12 / 141,809(<0.01)$ & $1.84(0.56-6.03)$ \\
\hline
\end{tabular}

Abbreviations: $\mathrm{Cl}=$ hazard ratio; $\mathrm{HR}=$ hazard ratio.

a Significant results.

${ }^{\mathrm{b}}$ Age at first recorded diagnosis of epilepsy (and corresponding age in matched controls).

The risk of ASD was increased in all calendar periods, with the highest risk estimate among individuals diagnosed with epilepsy before 1986 (HR 15.76, 95\% CI 11.75-21.14) (table e-1 on the Neurology ${ }^{(B)}$ Web site at Neurology.org).

Estimates tended to increase when the exposure was restricted to $\geq 2$ records of epilepsy and outcome to $\geq 2$ records of ASD (table e-1).

To examine the temporal relationship between epilepsy and ASD, we studied the risk of ASD diagnosis occurring before epilepsy. In this analysis, we also found an increased occurrence of ASD prior to the epilepsy diagnosis (OR 4.56, 95\% CI 4.02-5.18).

The initial sample included 80,511 siblings and 98,534 offspring of individuals with epilepsy. When we excluded relatives of epilepsy patients with ASD and their controls and all relatives and controls with a diagnosis of epilepsy, 77,895 siblings and 98,458 offspring remained. Initially, there were $104,121 \mathrm{pa}-$ rents of patients with epilepsy. Of these, 100,154 were parents of epilepsy patients without a diagnosis of ASD.

ASD was more common in siblings of individuals with epilepsy than in control siblings, with an excess risk of some 50\% (table 3). In siblings of individuals with epilepsy (including those individuals with epilepsy who had ASD), there was a 1.62-fold increased risk of ASD (95\% CI 1.43-1.83), decreasing to 1.34 when those siblings whose index individuals with epilepsy also had an ASD diagnosis were excluded (95\% CI 1.16-1.55) (table 3).

The risk of ASD was similar in female and male siblings (table e-2). No significant differences in ASD risk were found based on the sex of the index individual with epilepsy (table e-2).

Offspring of individuals with epilepsy, in comparison with control offspring, were at increased risk of ASD (HR 1.64, 95\% CI 1.46-1.84).

If the mother had epilepsy, the risk of ASD in the offspring was slightly higher (HR 1.91, 95\% CI 1.63-2.23) than if the father had epilepsy (HR $1.38,95 \%$ CI 1.16-1.64) (table 3 and table e-3).

We analyzed the occurrence of ASD in parents of individuals with epilepsy but restricted our data analysis to index individuals diagnosed with epilepsy from 1997 and onwards. We used this strategy since the ASD diagnosis is only recorded since 1987 (start of ICD-9 in Sweden). We found no increased risk of

Table 3 Risk of autism spectrum disorder (ASD) in the siblings and offspring of individuals with epilepsy

\begin{tabular}{lll}
$\begin{array}{l}\text { No. }(\%) \text { of ASD cases } \\
\text { in patients with epilepsy }\end{array}$ & $\begin{array}{l}\text { No. (\%) of ASD cases } \\
\text { in controls }\end{array}$ & HR $(95 \%$ CI) \\
\hline $336 / 80,511(0.4)$ & $1,067 / 408,677(0.3)$ & $1.62(1.43-1.83)^{a}$ \\
$392 / 98,534(0.4)$ & $1,188 / 495,698(0.2)$ & $1.64(1.46-1.84)^{a}$ \\
& & \\
$242 / 77,895(0.3)$ & $996 / 407,546(0.2)$ & $1.34(1.16-1.55)^{a}$ \\
$389 / 98,458(0.4)$ & $1,187 / 495,597(0.2)$ & $1.64(1.46-1.84)^{a}$ \\
$222 / 48,139(0.5)$ & $581 / 242,624(0.2)$ & $1.91(1.63-2.23)^{a}$ \\
$167 / 50,319(0.3)$ & $606 / 252,973(0.2)$ & $1.38(1.16-1.64)^{a}$
\end{tabular}

Abbreviations: $\mathrm{Cl}=$ hazard ratio; $\mathrm{HR}=$ hazard ratio

a Significant results. 
ASD in parents of individuals with epilepsy (HR $0.70,95 \%$ CI $0.33-1.48$ ), but ASD was very uncommon in both index and control parents.

DISCUSSION This nationwide cohort study of more than 85,000 individuals with epilepsy demonstrated a 10-fold increased risk of future ASD, as well as an increased future risk of ASD in first-degree relatives. Of note, there was a bidirectional relationship between epilepsy and ASD, i.e., individuals with epilepsy were also at increased risk of having an earlier ASD diagnosis (OR 4.56).

Our data on the risk of ASD in individuals with epilepsy are consistent with earlier studies, ${ }^{3-5}$ although previous risk estimates have varied substantially. One American study based on hospital health care data reported an OR of more than 22 for the association between ASD and epilepsy. ${ }^{3}$ Two British studies combined included 26 patients with both epilepsy and ASD. ${ }^{4,5}$ Our risk estimates were based on more than 1,300 patients with both epilepsy and ASD. This large sample size yielded high statistical power and narrower CIs (for instance, the $95 \%$ CI for future ASD was between 9.55 and 11.53). The large number of study participants also allowed for the examination of specific strata.

We found a significantly higher risk of ASD in women with epilepsy. ASD more often remains undiagnosed in female patients and we speculate that a diagnosis of epilepsy increases the chances of undiagnosed ASD being detected. Other data suggest an increased occurrence of epilepsy in female patients with ASD compared with male patients with ASD. ${ }^{6,9,13}$ This may be especially so in female patients with comorbid intellectual disability. ${ }^{9}$ In the Amiet et al. ${ }^{13}$ meta-analysis, the male:female ratio of autism comorbidity with epilepsy was $2: 1$, whereas the male: female ratio of autism without epilepsy was 3.5:1. We had no information on intellectual disability.

The risk of future ASD was significantly increased in childhood-onset epilepsy. Many types of childhood epilepsy are part of broader neurodevelopmental disorders that are known to be associated with neuropsychiatric disorders such as ASD. ${ }^{14,15}$ Epilepsy with onset later in life has etiologies (e.g., stroke and dementias) different from childhood-onset epilepsy. This might explain the lower relative risk of ASD in older individuals with epilepsy.

Our study shows increased risks of ASD in both the offspring and siblings of patients with epilepsy.

The risk of ASD in the offspring of female patients with epilepsy was high (HR 1.91, 95\% CI 1.63$2.23)$; in the offspring of male patients, the risk was somewhat lower (HR 1.38, 95\% CI 1.16-1.64). The high risk of ASD in the offspring of female patients with epilepsy may be due to fetal exposure to antiepileptic medication in utero. Studies have shown that use of valproic acid during pregnancy is associated with increased risk of ASD in offspring. ${ }^{16,17}$

In contrast to our results, a Danish case-control study reported that epilepsy was not more common in parents of individuals with ASD than in control parents. ${ }^{18}$ In our study, parents of individuals with epilepsy had no increased risk of ASD. However, the absolute risk of ASD was low in the parental group (independently of epilepsy in the index individual or not), probably due to factors concerning general functioning or the possibility to establish a family when an individual has autism. A British study of 150 individuals with autism found no increased risk of epilepsy, but of a broader autism phenotype in their relatives, suggesting that ASD of a genetic origin contributes to an increased risk of epilepsy. ${ }^{19}$

Both epilepsy and ASD were identified through diagnoses in the nationwide Swedish Patient Register, a register that is almost complete for inpatient data and that includes hospital-based outpatient data since 2001. Research suggests that the majority of individuals with epilepsy are recorded in the Swedish Patient Register, ${ }^{20,21}$ although epilepsy onset may precede first entry into a hospital-based register. Incomplete recording for ASD prior to ICD-9 and year 1987 (when the diagnosis was entered in Sweden) is unlikely to have affected our relative risks since controls were matched for calendar year. The validity of the epilepsy diagnosis is fairly high and the positive predictive value for epilepsy in the Swedish Inpatient Register (main part of the Swedish Patient Register) has been estimated at $79 \% .{ }^{21}$ A study in Denmark (a country with similar national register-based health care to Sweden) found a positive predictive value for epilepsy of $81 \%$ and for any seizure disorder of $89 \% .^{22}$ According to the Danish validation study of the ICD codes for different specific epilepsies in Health Care Registers, their predictive value is low in the subgroups. ${ }^{22}$ The occurrence of the specific epilepsy diagnosis varied in different studies of individuals with ASD and comorbid epilepsy, primarily generalized between $14 \%$ and $24 \%$, partial onset between $30 \%$ and $44 \%$, and the dominating group had unspecified epilepsy (G40.9). 6,9,23 We have therefore not analyzed the different epilepsy codes separately.

Risk estimates increased somewhat when we restricted our exposure to individuals with $\geq 2$ records of epilepsy and our outcome to $\geq 2$ records of ASD.

A Swedish study recently found that $96 \%$ of all ASD cases identified in the Swedish Patient Register could be confirmed through patient chart examination. ${ }^{24}$

One limitation of our study is our lack of data on medication. Exposure to certain antiepileptic 
medication may influence fetal development and could potentially increase the risk of ASD in the offspring. Yet contradicting this latter hypothesis as a main cause is evidence that the risk of ASD was similar in the offspring and siblings of patients with epilepsy.

The most likely explanation of our findings is shared pathophysiologic, possibly genetic, mechanisms, some influencing the balance between excitation and inhibition. ${ }^{14,25}$ Tuberous sclerosis complex and other rarer syndromes where ASD and epilepsy both occur are examples where different etiologies lead to both ASD and epilepsy. There are most likely different factors influencing the core mechanisms behind epilepsy and ASD (their core mechanisms being close or similar), bringing the patient to a tipping point where one or both disorders develop. It has been suggested that the 2 diseases share risk factors or heritability, ${ }^{14,26}$ which is consistent with our findings of a bidirectional temporal relationship, reinforcing the suggestion that seizures are not the cause of ASD.

It is not likely that the inability to adjust for intellectual disability in our analyses regarding ASD in relatives of individuals with epilepsy has changed our results significantly as we analyzed the risk in relatives without epilepsy of individuals without comorbid ASD.

Several other studies have reported bidirectional relationships between psychiatric disease and epilepsy. ${ }^{27,28}$ A systematic review and meta-analysis found a 7.8-fold increased risk of psychosis in individuals with epilepsy. ${ }^{29}$ Additional to shared genetics, patients with epilepsy and ASD have frequent contacts with health care. Thus, we cannot rule out that surveillance bias (also present in relatives) has contributed to the excess risks witnessed in our study.

Individuals with epilepsy are at increased risk of ASD, particularly if epilepsy appears in childhood or if the individuals are female. The high risk of ASD should have unique implications for the health care of patients with epilepsy. This group of patients should be screened for ASD, and if ASD is suspected, assessed for a diagnosis of the disorder. Our finding that ASD is also more common in siblings and offspring of individuals with epilepsy suggests shared etiology and an overlapping inheritance.

\section{AUTHOR CONTRIBUTIONS}

Heléne Sundelin: study concept and design, analysis and interpretation of data, drafting and revising the manuscript. Henrik Larsson: study concept and design, acquisition of data, critical revision of manuscript for intellectual content. Paul Lichtenstein: study concept and design, acquisition of data, critical revision of manuscript for intellectual content. Catarina Almqvist: critical revision of manuscript for intellectual content. Christina M. Hultman: critical revision of manuscript for intellectual content. Torbjörn Tomson: study concept and design, critical revision of manuscript for intellectual content. Jonas F. Ludvigsson: study concept and design, analysis and interpretation of data, critical revision of manuscript for intellectual content, supervision.

\section{STUDY FUNDING}

H.L.: The Swedish Research Council (2014-3831) and the Swedish Initiative for research on Microdata in the Social and Medical Sciences (SIMSAM), framework grant no. 340-2013-5867. P.L.: The Swedish Research Council for Health, Working Life and Welfare (project 20121678) and the Swedish Research Council (2011-2492). C.A.: The Swedish Research Council through the Swedish Initiative for research on Microdata in the Social and Medical sciences (SIMSAM), framework grant no. 340-2013-5867. T.T.: Stockholm County Council (ALF). None of the funders had any role in the design or conduct of the study; collection, management, analysis, or interpretation of the data; or preparation, review, or approval of the manuscript.

\section{DISCLOSURE}

H. Sundelin reports receiving travel costs for expert meeting from Cyberonics. H. Larsson has served as a speaker for Eli-Lilly and has received a research grant from Shire (both outside the submitted work). P. Lichtenstein, C. Almqvist, and C. Hultman report no disclosures relevant to the manuscript. T. Tomson is a full-time employee of Karolinska Institutet, has been associate editor of Epilepsia and is currently associate editor for Epileptic Disorders, has received speaker's honoraria to his institution from Eisai, UCB, and Actavis, and received research support from Stockholm County Council, CURE, GSK, UCB, Eisai, Bial, and Novartis. J. Ludvigsson reports no disclosures relevant to the manuscript. Go to Neurology.org for full disclosures.

Received October 22, 2015. Accepted in final form March 3, 2016.

\section{REFERENCES}

1. Fisher RS, van Emde Boas W, Blume W, et al. Epileptic seizures and epilepsy: definitions proposed by the International League Against Epilepsy (ILAE) and the International Bureau for Epilepsy (IBE). Epilepsia 2005;46: 470-472.

2. Lai MC, Lombardo MV, Baron-Cohen S. Autism. Lancet 2014;383:896-910.

3. Selassie AW, Wilson DA, Martz GU, Smith GG, Wagner JL, Wannamaker BB. Epilepsy beyond seizure: a population-based study of comorbidities. Epilepsy Res 2014;108:305-315.

4. Reilly C, Atkinson P, Das KB, et al. Neurobehavioral comorbidities in children with active epilepsy: a populationbased study. Pediatrics 2014;133:e1586-e1593.

5. Rai D, Kerr MP, McManus S, Jordanova V, Lewis G, Brugha TS. Epilepsy and psychiatric comorbidity: a nationally representative population-based study. Epilepsia 2012; 53:1095-1103.

6. Mouridsen SE, Rich B, Isager T. Epilepsy in individuals with a history of Asperger's syndrome: a Danish nationwide register-based cohort study. J Autism Dev Disord 2013;43:1308-1313.

7. Amiet C, Gourfinkel-An I, Laurent C, et al. Does epilepsy in multiplex autism pedigrees define a different subgroup in terms of clinical characteristics and genetic risk? Mol Autism 2013;4:47.

8. Kohane IS, McMurry A, Weber G, et al. The comorbidity burden of children and young adults with autism spectrum disorders. PLoS One 2012;7:e33224.

9. Jokiranta E, Sourander A, Suominen A, TimonenSoivio L, Brown AS, Sillanpaa M. Epilepsy among children and adolescents with autism spectrum disorders: a population-based study. J Autism Dev Disord 2014; 44:2547-2557.

10. Ludvigsson JF, Andersson E, Ekbom A, et al. External review and validation of the Swedish national inpatient register. BMC Public Health 2011;11:450. 
11. Ludvigsson JF, Otterblad-Olausson P, Pettersson BU, Ekbom A. The Swedish personal identity number: possibilities and pitfalls in healthcare and medical research. Eur J Epidemiol 2009;24:659-667.

12. Ludvigsson JF, Haberg SE, Knudsen GP, et al. Ethical aspects of registry-based research in the Nordic countries. Clin Epidemiol 2015;7:491-508.

13. Amiet C, Gourfinkel-An I, Bouzamondo A, et al. Epilepsy in autism is associated with intellectual disability and gender: evidence from a meta-analysis. Biol Psychiatry 2008; 64:577-582.

14. Lee BH, Smith T, Paciorkowski AR. Autism spectrum disorder and epilepsy: disorders with a shared biology. Epilepsy Behav 2015;47:191-201.

15. Andell E, Tomson T, Carlsson S, et al. The incidence of unprovoked seizures and occurrence of neurodevelopmental comorbidities in children at the time of their first epileptic seizure and during the subsequent six months. Epilepsy Res 2015;113:140-150.

16. Wood AG, Nadebaum C, Anderson V, et al. Prospective assessment of autism traits in children exposed to antiepileptic drugs during pregnancy. Epilepsia 2015;56:1047-1055.

17. Christensen J, Gronborg TK, Sorensen MJ, et al. Prenatal valproate exposure and risk of autism spectrum disorders and childhood autism. JAMA 2013;309:1696-1703.

18. Mouridsen SE, Rich B, Isager T. Epilepsy and other neurological diseases in the parents of children with infantile autism: a case control study. Child Psychiatry Hum Dev 2008;39:1-8.

19. Bolton PF, Carcani-Rathwell I, Hutton J, Goode S, Howlin P, Rutter M. Epilepsy in autism: features and correlates. Br J Psychiatry 2011;198:289-294.

20. Mattsson P, Tomson T, Eriksson O, Brannstrom L, Weitoft GR. Sociodemographic differences in antiepileptic drug prescriptions to adult epilepsy patients. Neurology 2010;74:295-301.

21. Nilsson L, Tomson T, Farahmand BY, Diwan V, Persson PG. Cause-specific mortality in epilepsy: a cohort study of more than 9,000 patients once hospitalized for epilepsy. Epilepsia 1997;38:1062-1068.

22. Christensen J, Vestergaard M, Olsen J, Sidenius P. Validation of epilepsy diagnoses in the Danish National Hospital Register. Epilepsy Res 2007;75:162-170.

23. Mouridsen SE, Rich B, Isager T. Epilepsy and other central nervous system diseases in atypical autism: a case control study. J Neural Transm 2011;118:621-627.

24. Idring S, Rai D, Dal H, et al. Autism spectrum disorders in the Stockholm Youth Cohort: design, prevalence and validity. PLoS One 2012;7:e41280.

25. Fakhoury M. Autistic spectrum disorders: a review of clinical features, theories and diagnosis. Int J Dev Neurosci 2015;43:70-77.

26. Cukier HN, Dueker ND, Slifer SH, et al. Exome sequencing of extended families with autism reveals genes shared across neurodevelopmental and neuropsychiatric disorders. Mol Autism 2014;5:1.

27. Adelow C, Andersson T, Ahlbom A, Tomson T. Hospitalization for psychiatric disorders before and after onset of unprovoked seizures/epilepsy. Neurology 2012;78:396-401.

28. Hesdorffer DC, Ishihara L, Mynepalli L, Webb DJ, Weil J, Hauser WA. Epilepsy, suicidality, and psychiatric disorders: a bidirectional association. Ann Neurol 2012; 72:184-191.

29. Clancy MJ, Clarke MC, Connor DJ, Cannon M, Cotter DR. The prevalence of psychosis in epilepsy: a systematic review and meta-analysis. BMC Psychiatry 2014;14:75.

\section{Look What's New at the 2016 AAN Fall Conference}

Visit AAN.com/view/fall to register for the 2016 AAN Fall Conference, set for October 14-16, at The Cosmopolitan of Las Vegas. This is your year-end destination for acquiring the latest clinical advances in key disease states, improving your practice's efficiency and bottom line, and earning up to $15.75 \mathrm{CME}$ credits.

\section{Look What's New!}

- All-inclusive registration rate offers greater value than ever

- Flexible meeting format lets you build your own tailored schedule and select the programs of most interest-while on the go on-site

- New Topics: Update in Stroke, AAN Leadership University Course: Challenges of Leadership in Private Practice, Headache Skills Workshop (pre-registration required) 